McElroy, T., \& Seta, J. J. (2004). On the other hand am I rational? Hemispheric activation and the framing effect.

Brain and Cognition, 55: 572-580. Published by Elsevier (ISSN: 0278-2626).

\title{
On the Other Hand Am I Rational? Hemispheric Activation and the Framing Effect
}

Todd McElroy and John J. Seta

\begin{abstract}
In recent decades the investigation of framing effects has become the foremost studied phenomenon of rational/irrational decision making. Two experiments were conducted to determine whether the functional specializations of the left and the right hemispheres would produce different responses to a traditional framing task. In Experiment 1, a behavioral task of finger tapping was used to induce asymmetrical activation of the respective hemispheres. In Experiment 2, a monaural listening procedure was used. In both experiments, the predicted results were found. Framing effects were found when the right hemisphere was selectively activated whereas they were not observed when the left hemisphere was selectively activated. The results are interpreted in light of a dual-processing approach of decision-making.
\end{abstract}




\section{INTRODUCTION}

In recent decades the issue of framing has become of paramount interest in many different areas of decision making (see Kuhberger, 1998 for review). Framing refers to situations where participants are presented with options that are framed either positively or negatively and each of the options has the same expected outcome. In a typical risky choice framing task, participants are forced to make a choice between a risk-free and a riskseeking option (Levin, Schneider, \& Gaeth, 1998). The typical finding from a risky choice decision task is that people tend to choose the risk-free option when the problem is framed positively and the risk-seeking option when the problem is framed negatively. Because most people demonstrate this reversal of preference, basing their decision on the frame rather than the expected outcome, the framing effect has become one of the most widely tested examples of irrationality in decision making.

\subsection{Framing research}

Seminal work by Kahneman and Tversky (1979) sparked interest into research investigating the risky choice framing paradigm. Kahneman and Tversky put forth prospect theory as a theoretical model for explaining why these types of gain/loss framing effects occur. Since that time researchers have used many different variations of the risky choice framing task.

However, the foremost studied example of framing is that of the Asian disease problem (Tversky \& Kahneman, 1981). In the Asian disease scenario, participants are asked to consider the following problem:

Imagine that the US is preparing for the outbreak of an unusual Asian disease, which is expected to kill 600 people. Two alternative programs to combat the disease have been proposed. In the gains condition, participants are faced with a risk-free option (200 people will be saved) and a risk-seeking option (a one-third probability that 600 people will be saved and a two-thirds probability that no people will be saved). Similarly, participants in the losses condition are also faced with a risk-free (400 people will die) and a risk-seeking option (one-third probability that nobody will die and a two-thirds probability that 600 people will die).

The finding for this traditional framing task is that participants in the gains condition tend to choose the risk-free option (200 people saved) whereas participants in the losses condition tend to choose the risk-seeking 
option (one-third probability that nobody will die and a two-thirds probability that 600 will die). This type of decision choice is considered irrational because the expected outcome of lives between the risk-free and riskseeking option is the same in both the gains (200 saved) and losses (400 die) conditions. Therefore, there should be no difference between the two conditions in risk-free or risk-seeking choices. The finding that such a reversal in choice exists attests to the fact that participants are relying upon the frame to make their choice rather than the expected outcome. A reliance on the frame rather than the expected outcome is considered irrational by traditional models of rational decision-making.

\subsection{Prospect theory}

Most theoretical models of decision-making focus on a strictly logical approach, relying only on the net assets of the outcomes (e.g., Machina, 1982; Savage, 1954; Von Neumann \& Morgenstern, 1944). Prospect theory, on the other hand, focuses on changes from the status quo and is sensitive to relative differences of gains and losses. And it is this evaluative sensitivity to gains and losses that provides the basis for interpreting framing effects. According to prospect theory (Kahneman \& Tversky, 1979, 1984; Tversky \& Kahneman, 1981), the decision making process is dichotomous, consisting of two phases. The initial phase involves coding the options as gains or losses relative to a neutral reference point. In the subsequent phase the subjective values and weighted probabilities for each of the options are evaluated in light of the prior editing phase. The options are then contrasted relative to each other. Framing effects in the evaluation phase are depicted by the S-shaped value function. The shape of this function is concave for gains and convex for losses. This depicts prospect theory's prediction of risk-aversion for gains and risk-seeking for losses.

\subsection{Framing limitations}

Researchers investigating framing effects have supported its potency across many different areas of research including medical decisions (e.g., McNeil, Pauker, Sox, \& Tversky, 1982; O'Connor, Pennie, \& Dales, 1996), monetary decisions (Fagley \& Miller, 1997), and taxes (Highhouse \& Paese, 1996). The finding that most people tend to rely upon the frame rather than the expected outcome across these different areas attests 
to the robustness of the effect. Although framing effects have been found in many different areas of research, an almost equal number of studies within these areas have reported weak or non-existent framing effects (e.g., Bless, Betsch, \& Franzen, 1998; Fagley \& Miller, 1997; O'Connor et al., 1996). Therefore, across many domains, the framing effect appears to be limited by certain cognitive and contextual boundaries.

Recently, research by McElroy and Seta (2003) has demonstrated that both cognitive and contextual factors can determine whether framing effects are evinced in a decision-making task. McElroy and Seta demonstrated that when individuals either had a personality disposition toward or were induced through the self-relevance of the decision task to engage a framing task more analytically, typical framing effects were not found. Conversely, when individuals had a personality disposition toward or were induced through relevance to engage a framing task holistically, the typical framing effects were found.

McElroy and Seta interpret these results as evidence for a dual analytic-holistic processing perspective. That is, when individuals engage a decision task with an analytic processing style they rely upon a logical, systematic analysis of each of the options. Consequently, this type of analysis is especially sensitive to analytic information, such as the numeric potentiation of the alternatives, and relatively insensitive to contextual information. And because the alternatives are of exactly the same expected value, there should be no difference between the gains and losses conditions. Conversely, individuals who engage the same task with a holistic processing style are especially sensitive to contextual cues and relatively insensitive to analytic information. Because of this, holistically focused individuals are sensitive to the frame, and the predicted framing effects occur. Following this line of research, the current studies were designed to test the analytic/holistic analysis at the level of hemispheric specialization. Specifically, we wanted to explore whether the functional specializations of the respective hemispheres would produce different types of responses to a traditional framing task.

\subsection{Hemispheric approach}

The findings of hemispheric specialization indicate that the two hemispheres engage in two very different forms of information processing. Research on the activities 
of the left hemisphere reveal a mode of processing that is logical and analytical (e.g., Bergson, 1965;

Cacioppo, Petty, \& Quintanar, 1982; Ornstein, 1977). Numerous studies have shown that the left hemisphere's mode of processing consistently breaks information down into different analyzable parts. The information is first reduced to a discreet form and then systematically analyzed in an orderly and logical fashion. In so doing, the option with the greatest numeric weight is systematically chosen.

The right hemisphere, on the other hand, is often associated with being "holistic" in nature (Kitchens, 1991; Levy, 1974). Recent research investigating hemispheric specializations however, have provided a more in-depth analysis of right hemispheric processing and thus, a more definitive explanation of this hemisphere's "holistic" style. For example, Brownell and colleagues (e.g., Brownell, Carroll, Rehak, \& Wingfield, 1992; Brownell, Pincus, Blum, Rehak, \& Winner, 1997) have investigated the role of the right hemisphere in comprehending language and suggest that the right hemisphere has the role of referencing terms within which conversations are understood. Cues such as status, familiarity, and formality serve as terms of reference that the right hemisphere uses to properly orient individuals in a conversational setting.

Drawing upon findings from many different areas of research, Ornstein (1997) proposes that the right hemisphere plays the primary role in understanding context. It uses cues within the context to "weave" together meaning from encountered stimuli. Although Ornstein did not directly discuss the role of framing, it seems logically consistent that the right hemisphere should be particularly sensitive to the frame in which information is presented. Therefore, in accordance with this line of reasoning, we view the role of the right hemisphere as one of contextual referencing, one that is relatively less involved in problem analysis and relatively more involved in determining the framework within which the information will be analyzed. Specifically, we see the right hemisphere fitting encountered information into a referenced frame within which the problem is analyzed and meaning is derived. Thus, it's "holistic" nature may lie in its ability to engage in contextual referencing. 


\subsection{Contralateral hemispheric activation}

One particular phenomenon that has grown out of the hemispheric research deals with activation of the hemisphere opposite or contralateral from the side of the body (corporal hemispace) where attention is being directed. For example, when attention is directed toward the right side of the body, the left hemisphere should become relatively more activated and vice versa. The beginning of this phenomenon has its roots in animal research (e.g., Myers, 1956; Van Wagenen \& Herren, 1940) but its importance for understanding human behavior was not recognized until the work of Kinsbourne (1970). In his activation-orientation hypothesis, Kinsbourne suggests that asymmetrical activation of either hemisphere can cause contralateral attention (e.g., eye or hand movements). Later research showed that the opposite could also occur. That is, lateral orientation of attention proved to be an effective means for selectively inducing relatively greater activation of the respective contralateral hemisphere (e.g., Bowers, Heilman, \& Van Den Abell, 1981; Malamed \& Larsen, 1977; Walker, Wade, \& Waldman, 1982). Researchers, relying upon this line of reasoning, have suggested that motor and sensory asymmetries found in the prenatal period may set the stage for later functional lateralization (e.g., Corballis, 1983; Kinsbourne \& Hiscock, 1983). This early development suggests a profound, early propensity for hemispatial motor and sensory connections to the contralateral hemisphere. Later work using EEG supported this view by demonstrating that lateral closed-eye movements are an indicator of relatively greater activity in the contralateral hemispheric (De Toffolo, Autret, Gaymaard, \& Degiovanni, 1992). This line of research has resulted in the use of lateralized attentional tasks as a means of selectively observing the influences of the respective hemispheres.

The predictions from this reasoning are straightforward. When attentional direction is aligned with a particular corporal hemispace, the contralateral hemisphere is relatively "hyperactive" and the ipsilateral hemisphere is relatively "hypoactive." As a consequence of this relationship, the relatively hyperactive hemisphere is especially prominent and influential, thereby allowing it to impinge its own particular specializations (or limitations) on the task at hand. The end result is that the relatively hyperactive hemisphere takes a leading role in information processing and predominately influences the eventual outcome of a given task. 


\subsection{Overview of Experiments 1 and 2}

Prior research has shown that when individuals process a decision task using the analytic style they are relatively insensitive to the way in which a problem is framed whereas when they process the same task with a holistic or contextual referencing style, framing effects are demonstrated (McElroy \& Seta, 2003). According to our hypothesis, individuals who are induced to have a relatively active left hemisphere should process the decision task using an analytic style and the framing of the task should have little or no influence on their decisions. The situation should be much different for those individuals who have a relatively active right hemisphere.

For these individuals, the way in which the task is framed should have a pronounced influence on their decisions and commonly observed framing effects should be obtained. In both of our experiments we tested our hypothesis by manipulating relative activation of the respective hemispheres. In Experiment 1, we utilized the simple behavioral method of finger tapping; in Experiment 2, we used the monaural listening procedure. Converging evidence will be obtained if the participants who were induced to have a relatively more active right hemisphere manifest customary framing effects whereas those participants who were induced to have a relatively more active left hemisphere do not.

\section{EXPERIMENT 1}

In Experiment 1, we induced the functional specializations of the respective hemispheres by capitalizing on the behavioral method of finger tapping. Consistent with prior assumptions (e.g., Bourgeois, Christman, \& Horowitz, 1998; Reuter-Lorenz, Kinsbourne, \& Moscovitch, 1990), we expect that when a participant's hemisphere is in a state of relatively greater activation, they will process a decision task with the thought style associated with the respective hemisphere. Therefore, although both hemispheres will quickly share information, the hemisphere that is relatively hyperactive should "taint" perceivers' thought processes with its own respective cognitive style.

The hemispheric activation method requires that attentional focus be directed on the side of the body opposite to the selected hemisphere. Researchers have used a variety of methods to manipulate an attentional focus on both the left and right side of the body or "corporal hemispace." These methods, according to Kinsbourne 
(1970) and others (Bowers et al., 1981; Malamed \& Larsen, 1977; Walker et al., 1982), should be a reliable indicator of contralateral hemispheric activation. Examples include conjugated lateral eye movements (Gur \& Reivich, 1980); language induced right bias (Bowers \& Heilman, 1976; Kimura, 1973; Kinsbourne, 1974), and hand positioning/movements (Parsons, Gabrieli, Phelps, \& Gazzaniga, 1998). We used a hand movement manipulation of finger tapping to induce attentional hyperactivation of the left and right hemispheres in Experiment 1.

\subsection{Method}

\subsubsection{Participants and design}

Forty-six female and five male undergraduate students participated in this study as part of a class project. The study was conducted while participants were in a group in a large room. Participants were divided into two relative hemispheric activation conditions (left/ right). This division was based on seating arrangement. The experimental material was then randomized so that each person had an equal probability of being represented in each of the experimental conditions. Selective hemispheric activation via finger tapping served as our 2 hemispheric activation (left/right) condition. The framing of the Asian disease problem in terms of either gains or losses served as our 2 framing (gain/loss) condition. 2.1.2. Behavioral hemispheric-activation manipulation Simple behavioral tasks, when hemispacially performed, normally serve to direct attention to the respective corporal hemispace. Therefore, one way of selectively inducing relative hemispheric activation is by having participants perform a simple behavioral task. One such example involves hand movements. Studies have shown that specific hand movements increase cerebral blood flow in the contralateral hemisphere (e.g., Cramer, Finkelstein, Schaechter, Bush, \& Rosen, 1999; Halsey, Blaunstenin, Wilson, \& Wills, 1979), which is an indicator of increased contralateral hemispheric activity. Therefore, in order to selectively induce relative hyperactivation of the respective hemispheres, we had participants continuously tap either their left or right fingers until after they had made their decision.

\subsubsection{Procedure}

Participants were separated into either the left hemisphere activation (right finger tapping) or right hemisphere 
activation (left finger tapping) condition based upon random seating arrangement. Stimulus materials were placed face down on the desk in front of each participant. Participants were first told that the experimenter was interested in their opinion regarding the information that they would be receiving. Participants were then told that during this experiment they were to tap their fingers. If they were seated on the left side (instructors view) they were told to tap their right finger and if they were seated on the right side (instructors view) they were told to tap their left finger. Participants were instructed that they should tap their respective fingers before they began, during the task, and to continue tapping until after they had made their decision. Participants were then told that they should indicate their choice on the paper located in front of them only after they had made their decision. Individual participation in the finger tapping conditions was monitored until the study was completed.1

The stimulus material contained the classic Asian disease problem developed by Tversky and Kahneman (1981), framed either in terms of gains or losses. After participants had made their decision, they were debriefed and all materials were collected.

\subsection{Results and discussion: Experiment 1}

Three participants were not included in our analysis because their ranking on our handedness questionnaire indicated that they were primarily left-handed. This questionnaire was adapted from Peters (1998). We used the 10 items that were shown in Peters (1998) factor analysis to be the best discriminators of handedness. These same questions were used for both studies 1 and 2 . We performed a nominal logistic v2 analysis on participants' decisions. This analysis revealed a main effect for gain/loss framing, $X^{2}(1, N=48)=6: 02, p<: 05$ as well as the predicted left/right hemisphere activation $x$ gain/loss framing interaction, $X^{2}(1, N=48)=$ $5: 19, p<.05 .2$ The decisions of participants in the left hemisphere activation (right finger tapping) condition were insensitive to the framing of the task. In this situation, the framing of options, either in terms of gains or losses, had no significant impact on whether participants chose the risk-averse option (option $A$ ) or the risk-seeking option (option B), $X^{2}(1, N=23)=.02 p>.8$ (see Table 1). Regardless of framing, there was a general risk-aversion trend; participants chose the risk-averse option more often than the risk-seeking option, $\mathrm{X}^{2}(1, \mathrm{~N}$ $=23)=8.67, p<.01$. 
Table 1

Number of risk-avoidant and risk-seeking choices as a function of hemispheric activation and framing

\begin{tabular}{|c|c|c|c|c|}
\hline \multirow[t]{3}{*}{ Framing } & \multicolumn{4}{|c|}{ Choices } \\
\hline & \multicolumn{2}{|c|}{ R isk-avoidant } & \multicolumn{2}{|c|}{ Risk-seeking } \\
\hline & $N$ & $\%$ total & $N$ & $\%$ total \\
\hline & \multicolumn{4}{|c|}{ Left hemisphere } \\
\hline Gains & 8 & 67 & 4 & 33 \\
\hline \multirow[t]{2}{*}{ Losses } & 7 & 64 & 4 & 36 \\
\hline & \multicolumn{4}{|c|}{ Right hemisphere } \\
\hline Gains & 12 & 92 & 1 & 08 \\
\hline Losses & 3 & 25 & 9 & 75 \\
\hline
\end{tabular}

The decisions of participants in the right hemisphere activation (left finger tapping) condition were especially sensitive to the gain/loss framing manipulation, $X^{2}(1, N$ $=25)=8.4, p<.01$. In the gains condition, participants demonstrated risk aversion by choosing the risk-averse option more often (92\%), X2(1, N = 13) = 56.7, $\mathrm{p}<.001$ than the risk-seeking option whereas an opposite pattern emerged in the losses condition; participants in this condition chose the risk-seeking option more often than the risk-averse option (75\%), $X^{2}(1, N=12)=13.18, p<.001$. These results suggest that framing effects can be dependent upon the particular hemispheric processing style that an individual uses when performing a decision task. When the right hemisphere was induced to have relatively greater activation, it impinged its processing style on the decision task and participants' decisions were heavily influenced by contextual cues. Consequently, participants' decisions were influenced by the way in which the decision was framed. However, when the left hemisphere was induced to have relatively greater activation, it impinged its own analytic processing style and framing effects were weak or non-existent.

\section{EXPERIMENT 2}

In Experiment 1, our decision-making task was relatively simple. Consequently, its successful completion did not require large amounts of cognitive capacity. This is important because some studies (e.g., Low \& Rebert, 1978) have found that tasks requiring large amounts of cognitive capacity can place a high processing load on their respective contralateral control centers and as a result, the opposite hemisphere "picks-up" the processing 
of the task. For example, left finger tapping in a complex task could overload the right hemisphere leading the left hemisphere to dominate the processing of the task. Because the task used in Experiment 1 was relatively simple, it is extremely unlikely that our behavioral manipulation of finger tapping overloaded the contralateral hemisphere. Nevertheless, in Experiment 2, we used a monaural listening procedure to provide a further test of our hypothesis concerning the role that hemispheric style plays in decision-making.

The monaural listening procedure is passive and effortfree in that it induces lateral orientation of attention without a behavioral component (see Henry, 1983 for review). Therefore, it not only serves to induce relative activation of a particular hemisphere, it also allows the activated hemisphere freedom from cognitive load. Consequently, it allows the hemisphere that is activated to impinge its particular style of processing on the task (e.g., Bourgeois et al., 1998; De Toffolo et al., 1992; Drake \& Seligman, 1989). Therefore, even though both hemispheres are involved in the task, the hemisphere that is selectively activated should taint the process with its own particular processing style. Experiment 2 was designed with these considerations in mind.

\subsection{Method}

\subsubsection{Participants and design}

Forty-two female and six male participants took part in the study. Participants received credit for their participation as part of their class research requirement.

The design of this study was similar to that used in Study 1 . We employed a 2 (side of aural presentation: left vs. right) $\times 2$ (framing of outcome: gains vs. losses) between-participants factorial design. In each condition, participants were asked to choose between a risk-seeking and a risk-averse option.

\subsubsection{Monaural listening procedure}

In the monaural listening procedure stimulus material is presented monaurally to either the left or right ear. This technique has been shown to be a very reliable means for investigating hemispheric asymmetries (e.g., Drake, 1987, 1993; Drake \& Crow, 1989; Henry, 1983; Hines \& Martindale, 1974; Hiscock, Hampson, Wong, 
\& Kinsbourne, 1985; Hughes \& Zimba, 1985; Morais \& Bertelson, 1975) and consistently shows initial enhancement of the contralateral hemisphere. Because this technique is designed to produce directional shifts in attention, the critical component is not ear-of-entry. Rather, the critical component is that attention is directed toward the corporal hemispace contralateral to the targeted hemisphere. Therefore, as long as hemispacial attention is maintained, hyperactivation of the contralateral hemisphere should occur (e.g., De Toffolo et al., 1992; Tressoldi \& Cusumano, 1992). The consequential result, then, should be that the hemisphere that is contralateral to ear of entry should be hyperactive and impinge its particular thinking style on the decision task.

\subsubsection{Procedure}

Participants were seated separately in a room. The number of participants in each session ranged from 2 to 4 and each group was randomized so that each participant had an equal chance of being represented in each condition. The room was partitioned so that participants could not see one another throughout the study. Participants were given a set of typed instructions and told to raise either their right or left hand (depending upon which hemispheric presentation condition they were in) after they had finished reading. This set of instructions informed participants that they would be presented with a situation and that they would be asked their opinion regarding it. They also were told that information would be presented to them aurally and that, because context can sometimes influence opinions, they should look in the direction of the mark posted on the wall throughout the remainder of the experiment. The mark was posted either on the left (hemisphere presentation) or right side of the room (left hemisphere presentation). Having participants look in a particular direction is consistent with the procedure used by Drake (1987). The action of looking toward the right can facilitate activation of the left hemisphere, whereas the action of looking toward the left can facilitate activation of the right hemisphere (e.g., Galin \& Ornstein, 1974; Kinsbourne, 1974). Presentation for the remainder of the study was in the form of the experimenter's voice presented monaurally via headphones to either the left or right ear (e.g., Drake, 1993; Hiscock et al., 1985). All participants were presented monaurally with the Asian disease scenario (e.g., Tversky \& Kahneman, 1981) framed as either gains or losses. They were then instructed that after they made their decision, they were to 
indicate their choice on the piece of paper located in front of them.

Table 2

Number of risk-avoidant and risk-seeking choices as a function of hemispheric activation and framing

\begin{tabular}{|c|c|c|c|c|}
\hline \multirow[t]{3}{*}{ Framing } & \multicolumn{4}{|c|}{ Choices } \\
\hline & \multicolumn{2}{|c|}{ Risk-avoidant } & \multicolumn{2}{|c|}{ Risk-seeking } \\
\hline & $N$ & $\%$ total & $N$ & $\%$ total \\
\hline & \multicolumn{4}{|c|}{ Left hemisphere } \\
\hline Gains & 8 & 73 & 3 & 27 \\
\hline \multirow[t]{2}{*}{ Losses } & 7 & 64 & 4 & 36 \\
\hline & \multicolumn{4}{|c|}{ Right hemisphere } \\
\hline Gains & 9 & 82 & 2 & 18 \\
\hline Losses & 1 & 09 & 10 & 91 \\
\hline
\end{tabular}

\subsection{Results and discussion: Experiment 2}

Four participants were excluded from the analysis because their score on our handedness questionnaire (Peters, 1998) indicated that they were primarily left-handed. We performed a 2 (hemispheric presentation) x 2 (gain/loss) nominal logistic v2 analysis of participants decisions. This analysis revealed a main effect for framing $X^{2}(1, N=44)=6.98, p<.01$. More importantly, it also revealed the predicted hemisphere presentation (left versus right)_framing (gain versus loss) interaction, $X^{2}(1 ; \bar{N}=44)=4.47, p<.05$ (see Table 2).3 To decompose this interaction, we performed a nominal logistic v2 analysis on participants' decisions in each of the two hemisphere conditions. According to our predictions, participants in the left hemisphere condition should engage the information with a predominantly analytic processing style and as a result they should be relatively insensitive to the way in which the problem is framed. Participants in the right hemisphere condition, however, should engage the information with a very different processing style, referencing the context for the frame in which the information is presented. Consequently, their decisions should be heavily influenced by the way in which the problem is framed. As expected, we did not find a difference between the two framing conditions in the left hemisphere presentation (right ear) condition $\mathrm{X}^{2}(1, \mathrm{~N}=22)=.21, \mathrm{p}>.6$. Within this condition, regardless of framing, there was a general risk-aversion trend; participants made significantly 
more risk-averse choices than risk-seeking choices

$X^{2}(1, N=22)=12.06, p<.001$.

Predictions for the right hemisphere presentation condition were also upheld. We found a significant main effect for framing in the right-hemisphere (left ear) presentation condition, $X^{2}(1, N=22)=8.47, p<.01$. In the gains framing condition, participants chose the risk-averse option significantly more than the risk-seeking option, $X^{2}(1, N=11)=21.19, p<.001$ whereas in the losses framing condition, they chose the risk-seeking option more often than the risk avoidant option, $\mathrm{X}^{2}(1, \mathrm{~N}=11)=41.65, \mathrm{p}<.001$.

These results converge with those obtained in Experiment 1 and suggest that participants who process a decision task with a selectively activated right hemisphere are especially sensitive to the influence of contextual cues, such as the frame. Conversely, those participants who process a decision task with a selectively activated left hemisphere are not.

\section{GENERAL DISCUSSION}

The results of both studies provide evidence that when participants perform a traditional type of framing task their "rational" pattern of response differs depending upon whether they process the task with an activated right or activated left hemisphere. We found that when participants performed the framing task under conditions that selectively activated the right hemisphere, they were especially sensitive to how the problem was framed. As a result, these participants demonstrated the predicted framing effects. We observed a much different pattern of responses from participants who performed the framing task with a selectively activated left hemisphere. Under these conditions, participants were especially sensitive to the information contained in the task and relatively less influenced by contextual factors, such as the frame. As a result, little or no framing effects were observed.

\subsection{Hemisphere activation and risk-taking}

Although our main question dealt with how framing effects were influenced by differential hemispheric activation, our findings provide information about the "riskiness" of the respective hemispheres. Research by Drake (1985) and Drake and Ulrich (1992) found that 
participants who had a relatively activated or dominant left hemisphere tended to be more risk-seeking. In our studies, participants made a decision between two options framed either as gains or losses. When information was presented in this way, we saw that even though the decisions of individuals who had a relatively activated left hemisphere were not affected by the frame, their decisions did demonstrate an overall risk-averse tendency. On the other hand, the decisions of participants who had a relatively activated right hemisphere were affected by the frame; they demonstrated a risk-averse tendency when information was framed as a gain and a risk-seeking tendency when it was framed as a loss. Therefore, the relative "riskiness"of the two hemispheres was influenced by the way in which the problem was framed.

In the Drake studies, individuals who had a dominant or relatively activated left hemisphere were especially risk-seeking. However, the method of measuring risk was the subjective generation and interpretation of information; unlike in our studies, the information was not framed either as gains or losses and participants were not presented with a forced choice decision task. Therefore, one conclusion that can be drawn from these studies is that the tendency for individuals who have a relatively activated (or dominant) left hemisphere to be especially risk-seeking depends upon the method of risk assessment. Specifically, the pattern of riskiness may depend upon whether individuals are or are not influenced by the way in which the decision is framed. For example, when the task is not framed as either a gain or loss or when individuals are not influenced by the framing of the decision individuals who have a relatively activated (or dominant) left hemisphere may be especially risk-seeking. However, when individuals are influenced by the way in which the decision is framed (as a gain or loss) then results consistent with those obtained in the present studies should be observed.

It also may be the case that even when individuals make subjective estimations and interpretations, the "riskiness" of participants who have a relatively activated (or dominant) left hemisphere depends upon whether the information is framed in either positive (gains) or negative (losses) terms. If this possibility is correct then consistent with the findings of the present two studies, we should observe especially high levels of riskiness on the part of individuals who have relatively activated (or dominant) left hemispheres when information is framed in terms of gains but an opposite 
pattern should be observed when framed in terms of losses.

In closing, it should be emphasized that, although the "riskiness" issue provides direction for future research, it does not alter the primary message of the present paper. In both experiments, framing effects on a forced choice decision task were found when the right hemisphere was selectively activated. This was not the case for the left hemisphere.

Therefore, on the one hand, we see a right hemisphere that contributes to problem analysis by providing a framework for interpreting the problem. This framework is elicited by a process that we term contextual referencing. Specifically, the right hemisphere has special sensitivity to cues that provide information about the context in which the problem is embedded. These cues then allow the right hemisphere to reference a particular framework that is associated with the current decision.

On the other hand, we see the left hemisphere performing an analytic analysis of the information contained within the problem. We view the left hemisphere's role as one that initially breaks information down into discreet analyzable parts. The information is then systematically evaluated in an orderly and logical fashion.

Subsequent decisions are then especially sensitive to the strengths of the alternatives. Consequently, unlike the right hemisphere, we see the left hemisphere as relatively unaffected by contextual cues, such as the way in which the decision is framed.

\section{NOTES}

1. A possible limitation to our study might be participant's choice of seating arrangement. For example, Karev (2000) found that when indicating where they would sit on a movie theatre map, all participants showed a bias toward the right side. However, right handers tended to be relatively more biased. This suggests that those individuals with a dominant left hemisphere might have selected themselves to sit more in the right finger tapping condition relative to left handers. We do not feel this poses a problem to our study. In fact, this would actually work against our hypothesis. Further, research by Drake and Ulrich (1992) found no relationship between classroom seating and line bisecting - a measure of hemispheric predominance.

2. We performed an analysis to determine if there might be any effects for gender. The analysis revealed no effects $X^{2}(1, N=48)=<1$, 
$p>.8$. However, these results are limited by the small number of males in our study.

3. Similar to study 1 , we performed an analysis to determine if there might be any effects for gender. The analysis revealed no effects $X^{2}(1, N=44)=<1, p>.5$. These results are also limited by the small number of males in our study.

\section{REFERENCES}

Bergson, H. (1965). Duration and simultaniety. New York: BobbsMerrill.

Bless, H., Betsch, T., \& Franzen, A. (1998). Framing the framing effect: The impact of context cues on solutions to the Asian disease problem. European Journal of Social Psychology, 28, 287-291.

Bourgeois, M. J., Christman, S., \& Horowitz, I. A. (1998). The role of hemispheric activation in person perception: Evidence for an attentional focus model. Brain and Cognition, 38, 202-219.

Bowers, D., \& Heilman, K. M. (1976). Material specific hemispheric arousal. Neuropsychologia, 14, 123-127.

Bowers, D., Heilman, K. M., \& Van Den Abell, T. (1981). HemispaceVHF compatibility. Neuropsychologia, 19, 757-765.

Brownell, H. H., Carroll, J. J., Rehak, A., \& Wingfield, A. (1992). The use of pronoun anaphora and speaker mood in the interpretation of conversational utterances by right hemisphere brain-damaged patients. Brain and Language, 43, 121-147.

Brownell, H. H., Pincus, D., Blum, A., Rehak, A., \& Winner, E. (1997). The effects of right-hemisphere brain damage on patients' use of terms of personal reference. Brain and Language, 57, 60-79.

Cacioppo, J. T., Petty, R. E., \& Quintanar, L. R. (1982). Individual differences in relative hemispheric alpha abundance and cognitive responses to persuasive communications. Journal of Personality and Social Psychology, 43, 623-636.

Corballis, M. C. (1983). Human laterality. New York: Academic Press.

Cramer, S. C., Finkelstein, S. P., Schaechter, J. D., Bush, G., \& Rosen, B. R. (1999). Activation of distinct motor cortex regions during ipsilateral and contralateral finger movements. Journal of Neurophysiology, 81, 383-387. 
De Toffolo, B., Autret, A., Gaymaard, B., \& Degiovanni, E. (1992). Influence of lateral gaze on electroencephalographic spectral power. Elecroencephlography and Clinical Neurophysiology, 82, 432-437.

Drake, R. A. (1985). Lateral asymmetry of risky recommendations. Personality and Social Psychology Bulletin, 11, 409-417.

Drake, R. A. (1987). Conceptions of own versus others' outcomes: Manipulation by monaural attentional orientation. European Journal of Social Psychology, 17, 373-375.

Drake, R. A. (1993). Processing persuasive arguments: 2. Discounting of truth and relevance as a function of agreement and manipulated activation asymmetry. Journal of Research in Personality, 27, 184-196.

Drake, R. A., \& Crow, L. T. (1989). A role for hemispheric asymmetry in human behavioral variability. Pavlovian Journal of Biological Science, 24, 43-49.

Drake, R. A., \& Seligman, M. E. P. (1989). Self-serving biases in causal attributions as a function of altered activation asymmetry. International Journal of Neuroscience, 45, 199-204.

Drake, R. A., \& Ulrich, G. (1992). Line bisecting as a predictor of personal optimism and desirability of risky behaviors. Acta Psychologica, 79, 219-226.

Fagley, N. S., \& Miller, P. M. (1997). Framing effects and arenas of choice: Your money or your life? Organizational Behavior and Human Decision Processes, 71, 355-373.

Galin, D., \& Ornstein, R. (1974). Individual differences in cognitive style: Reflective eye movements. Neuropsychologia, 12, 367-376.

Gur, R. C., \& Reivich, M. (1980). Cognitive task effects on hemispheric blood flow in humans: Evidence for individual differences in hemispheric asymmetry. Brain and Language, 9, 78-92.

Halsey, J. H., Blaunstenin, U. W., Wilson, E. W., \& Wills, E. H. (1979). Regional cerebral blood flow comparison of right and left hand movement. Neuropsychology, 29, 21-28.

Henry, R. G. (1983). Monaural studies eliciting an hemispheric asymmetry: A bibliography II. Perceptual and Motor Skills, 56, 915-918.

Highhouse, S., \& Paese, P. W. (1996). Problem domain and prospect frame: Choice under opportunity versus threat. Personality and Social Psychology Bulletin, 22, 124-132. 
Hines, D., \& Martindale, C. (1974). Induced lateral eye movements and creative and intellectual performance. Perceptual and Motor Skills, 39, 153-154.

Hiscock, M., Hampson, E., Wong, S. C. P., \& Kinsbourne, M. (1985). Effects of eye movements on the recognition and localization of dichotic stimuli. Brain and Cognition, 4, 140-155.

Hughes, H. C., \& Zimba, L. D. (1985). Spatial maps of directed visual attention. Journal of Experimental Psychology: Learning, Memory, and Cognition, 10, 649-662.

Kahneman, D., \& Tversky, A. (1979). Prospect theory: An analysis of decision under risk. Econometrica, 47, 263-291.

Kahneman, D., \& Tversky, A. (1984). Choices, values, and frames. American Psychologist, 39, 341-350.

Karev, G. B. (2000). Cinema seating in right, mixed and left handers. Cortex, 36, 747-752.

Kimura, D. (1973). Manual activity in right-handers associated with speaking. Neuropsychologia, 11, 45-50.

Kinsbourne, M. (1970). The cerebral basis of lateral asymmetries in attention. Acta Psychologica, 33, 193-201.

Kinsbourne, M. (1974). The mechanisms of hemisphere asymmetry in man. In M. Kinsbourne \& W. L. Smith (Eds.), Hemispheric disconnection and cerebral function. Springfield, IL: Charles C. Thomas.

Kinsbourne, M., \& Hiscock, M. (1983). Development of functional lateralization of the brain. In P. H. Mussen (Ed.), Handbook of child psychology: Infancy and development psychobiology (Vol. II). New York: Wiley.

Kitchens, A. (1991). Left brain/right brain theory: implications for developmental math instruction. Review of Research in Developmental Education, 8, 20-23.

Kuhberger, A. (1998). The influence of framing on risky decision: A meta-analysis. Organizational Behavior and Human Decision Processes, $75,23-55$.

Levin, I. . P., Schneider, S. L., \& Gaeth, G. J. (1998). All frames are not created equal: A typology and critical analysis of framing effects. Organizational Behavior and Human Decision Processes, 76(2), 149-188. 
Levy, J. (1974). Psychobiological implications of bilateral symmetry. In S. Dimond \& S. Beaumont (Eds.), Hemispheric functions in human the brain. New York: Halstead Press.

Low, D. W., \& Rebert, C. S. (1978). Sex differences in cognitive/motor overload in reaction time tasks. Neuropsychologia, 16, 611-616.

Machina, M. J. (1982). "Expected Utility" analysis without the independence axiom. Econometrica, 50, 277-323.

Malamed, E., \& Larsen, B. (1977). Regional cerebral blood flow during voluntary conjugate eye movements in man. Acta Neurologica Scandinavica, 56, 530-531.

McElroy, T., \& Seta, J. J. (2003). Framing effects: An analyticholistic perspective. Journal of Experimental Social Psychology, 39, 610-617.

McNeil, B., Pauker, S. G., Sox, H. C., \& Tversky, A. (1982). On the elicitation of preferences for alternative therapies. New England Journal of Medicine, 306, 1259-1262.

Morais, J., \& Bertelson, P. (1975). Spatial position versus ear of entry as determinant of the auditory laterality effects: A stereophonic test. Journal of Experimental Psychology: Human Perception and Performance, 1, 243-262.

Myers, R. E. (1956). Function of corpus callosum in interocular transfer. Brain, 79, 358-363.

O'Connor, A. M., Pennie, R. A., \& Dales, R. E. (1996). Framing effects on expectation, decisions, and side effects experienced: The case of influenza immunization. Journal of Clinical Epidemiology, 49, 1271-1276.

Ornstein, R. E. (1977). The psychology of consciousness. Brace, Jovanovich, Chicago: Harcourt.

Ornstein, R. E. (1997). The right mind: Making sense of the hemispheres. New York: Harcourt Brace.

Parsons, L. M., Gabrieli, J. D. E., Phelps, E. A., \& Gazzaniga, M. S. (1998). Cerebrally lateralized mental representations of hand shape and movement. Journal of Neuroscience, 18, 6539-6548.

Peters, M. (1998). Description and validation of a flexible and broadly usable handedness questionnaire. Laterality, 3, 77-96.

Reuter-Lorenz, P. A., Kinsbourne, M., \& Moscovitch, M. (1990). Brain and Cognition, 12, 240-266. 
Savage, L. J. (1954). The foundations of statistics. New York: Wiley.

Tressoldi, P. E., \& Cusumano, S. (1992). Visual evoked potentials related to behavioral asymmetries during foveal attention in the two extrapersonal hemispaces. Brain and Cognition, 18, 125-137.

Tversky, A., \& Kahneman, D. (1981). The framing of decisions and the rationality of choice. Science, 221, 453-458.

Van Wagenen, W., \& Herren, R. (1940). Surgical division of commissural pathways in the corpus callosum. Archives of Neurology and Psychiatry, 44, 740-759.

Von Neumann, J., \& Morgenstern, O. (1944). Theory of Games and Economic Behavior. Princeton, New Jersey: Princeton University

Press.

Walker, E., Wade, S., \&Waldman, I. (1982). The effect of lateral visual fixation on response latency to verbal and spatial questions. Brain and Cognition, 1, 399-404. 